\title{
Primary Hyperparathyroidism Masquerading as Rickets
}

\author{
Kamran Amir Khan ${ }^{1}$ and Sameed Ullah Qureshi ${ }^{2}$ \\ Department of Internal Medicine 1 / Medicine ${ }^{2}$, Northwest General Hospital, Peshawar, Pakistan
}

\begin{abstract}
Primary hyperparathyroidism (PHPT) is common among adults. However, its incidence is rare among children associated invariably with delay in diagnosis. In children, it is caused by hyperplasia, adenoma or related hereditary disorders. Herein, a 17-year male with PHPT presented with rare skeletal manifestations of genu valgum and bilateral epiphyseal displacement of femur. Investigations done three years ago reported hypocalcemia and vitamin-D deficiency. He was diagnosed with rickets and received plenty of calcium and vitamin D supplements. Due to lack of clinical improvement following the supplementation, re-evaluation revealed hypercalcemia, elevated vitamin D levels and elevated parathyroid hormone (PTH). A 99mTc-sestamibi scan reported increased uptake in the left inferior lobe of the thyroid gland. Surgical removal of the parathyroid gland was done and histopathology revealed parathyroid adenoma. Rickets in the setting of PHPT can masquerade as PHPT. In a patient with rickets, hypercalcemia at presentation or following the calcium and vitamin supplementations, should warn the physicians to rule out PHPT.
\end{abstract}

Key Words: Primary hyperparathyroidism (PHPT), Hypercalcemia, Rickets, Vitamin D.

How to cite this article: Khan KA, Qureshi SU. Primary hyperparathyroidism masquerading as rickets. J Coll Physicians Surg Pak 2019; 29(9):891-4.

\section{INTRODUCTION}

Primary hyperparathyroidism (PHPT) occurs most commonly in 45 to 75 years of age and is rare among children and late adolescents. ${ }^{1}$ Skeletal deformities as a manifestation of PHPT are rare. Genu valgum is one of the skeletal deformities that can occur in children with PHPT.

Herein, we report a 17 -year male presenting with the classic clinical features of rickets. He was diagnosed with PHTP after laboratory analysis and sestamibi scan. Parathyroidectomy was performed; and the histopathology confirmed a parathyroid adenoma. The patient improved and showed resolution of all clinical features following the surgery.

\section{CASE REPORT}

A 17-year boy presented in May 2018 with a normal birth history and normal developmental milestones. History was significant for progressively worsening leg deformities and difficulty in walking. His symptoms started when he was 11 years old along with severe bone aches. The patient denied history of trauma, fall, bone fractures, vomiting, constipation, abdominal pain or neck swelling. The boy did not perform well in school and sports due to the limited physical activities including difficulty in walking. The past history was significant for multiple calcium and vitamin D supplements due to clinical

Correspondence to: Dr. Sameed Ullah Qureshi, Department of Medicine, Northwest General Hospital, Peshawar, Pakistan

E-mail: sameedqureshi92@gmail.com

Received: October 18, 2018; Revised: February 25, 2019;

Accepted: February 26, 2019 suspicion of rickets. Family history was insignificant for any kidney disease or malignancy including multiple endocrine neoplasia (MEN). He underwent osteotomy procedure for his left leg five months back. His previous records were traced, which showed a calcium level of $7.9 \mathrm{mg} / \mathrm{dl}$ and alkaline phosphatase of $867 \mathrm{U} / \mathrm{L}$.

On examination, he had generalised muscle wasting, tender ribs, lower limb showing valgus deformities of the knees bilaterally, eversion deformity of ankle joints, and flat feet with restricted movements (Figure 1). No other joint deformities were noted. According to WHO criteria, he had both low weight-for-age and height-for-age for his stage.

Previous radiographs depicted flaring, widening of the distal ends of femur and tibia along with splaying, cupping and fraying without epiphyseal closure. A postprocedure $X$ ray of left knee showed osteotomy changes (Figure 2). Laboratory investigations are given in Table I, and were suggestive of hypercalcemia, hypophosphatemia, raised parathyroid hormone (PTH) and vitamin $D$ levels. To exclude multiple endocrine disorders, thyroid function tests, follicle stimulety hormone (FSH), leutin hormone (LH), prolactin, amylase and baseline blood tests, serum electrolytes, and liver and renal profile were done, which all were normal except for raised alkaline phosphatase level. He received calcium and vitamin $D$ supplements over the course of last three years for the suspicion of rickets. His bone pains progressed, and he gradually developed chest pain. A chest $x$-ray showed ribbon ribs.

An ultrasound neck was performed to detect any nodule. Abdominal and pelvic radiological scans did not show any renal stone. $99 \mathrm{mTc}$-sestamibi scan showed an 
increased uptake of the left lobe of the thyroid gland, suggestive of left inferior parathyroid adenoma (Figure 3). The patient was given intravenous (IV) fluids, calcitonin and zoledronic acid for his hypercalcemia. Once his calcium levels improved, he had a left inferior parathyroidectomy and a parathyroid adenoma was detected on histopathology.

The patient was started on calcium and vitamin D supplementation after surgery to avoid hypocalcemia and

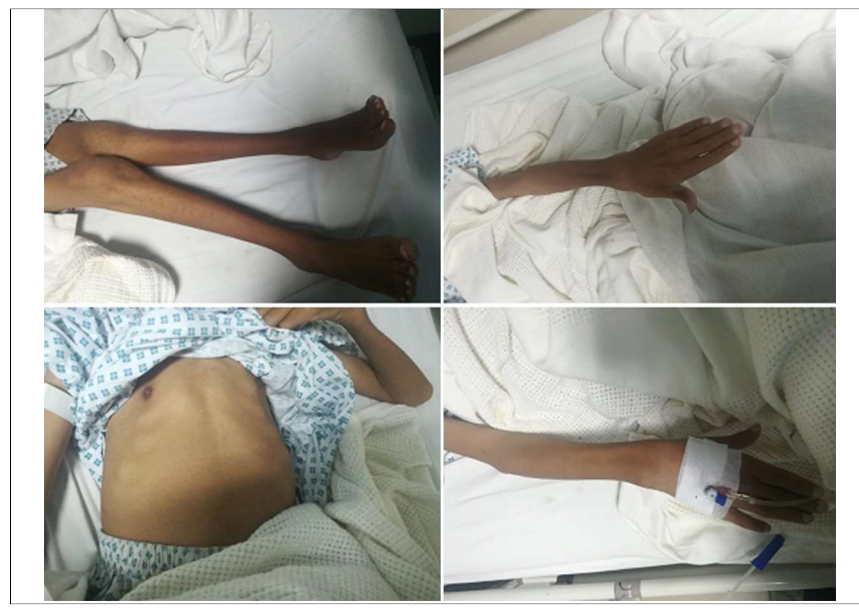

Figure 1: Bilateral valgus deformities of the knees, generalised muscle wasting, eversion deformity at ankle joints, flat feet and ribbon ribs.

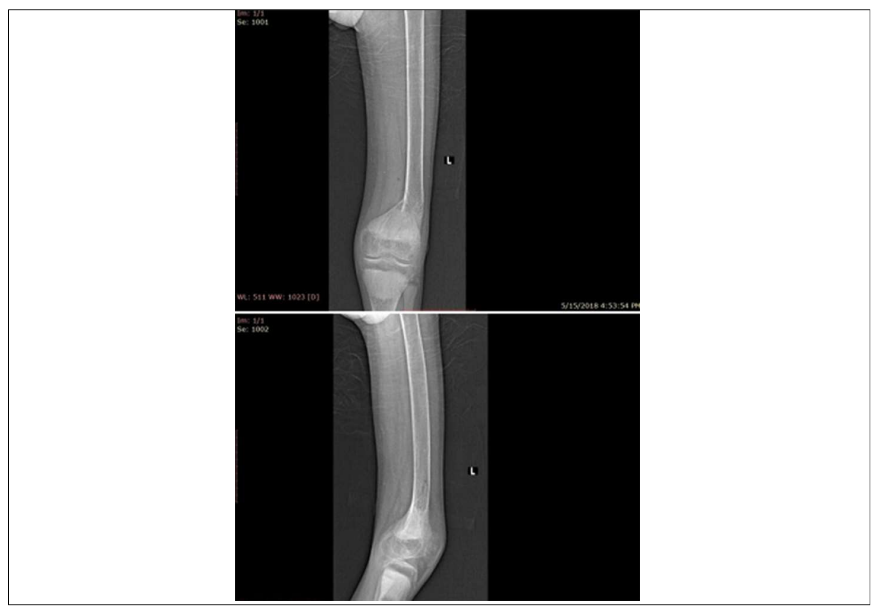

Figure 2: X-ray of the left knee showing postoperative changes. hungry bone disease. Another surgery for correction of limb deformity of another limb is planned following the improvement of quality of bone after treatment of PHPT. The patient is on regular follow-up and investigations; after two months, he revealed a normal calcium and parathyroid PTH. The patient is advised regular physiotherapy and showed a marked improvement in the lifestyle.

Follow-up scan at 2 months did not show tracer uptake (Figure 4), signifying successful removal of the adenoma.

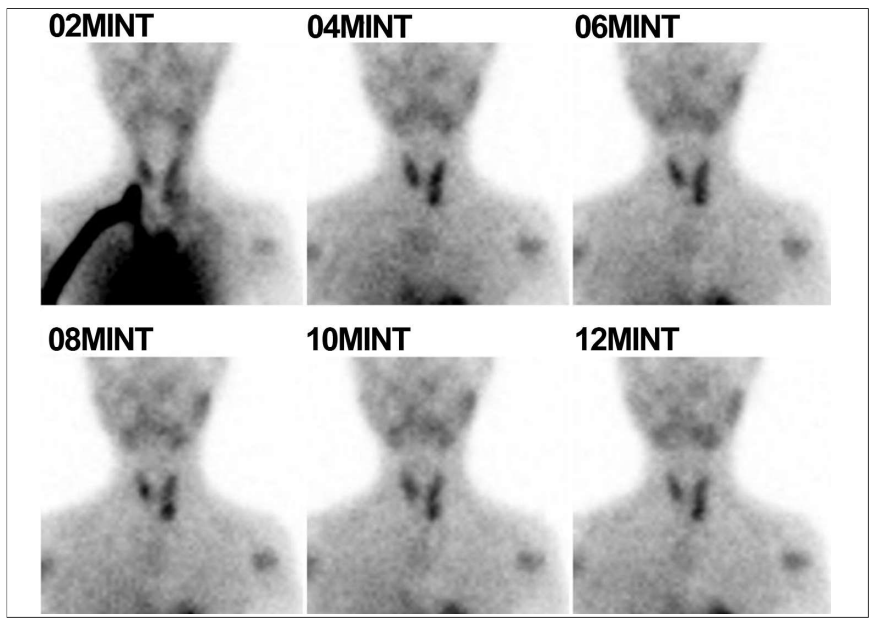

Figure 3: Scintigraphic evidence of parathyroid adenoma in the left inferior lobe of thyroid gland.

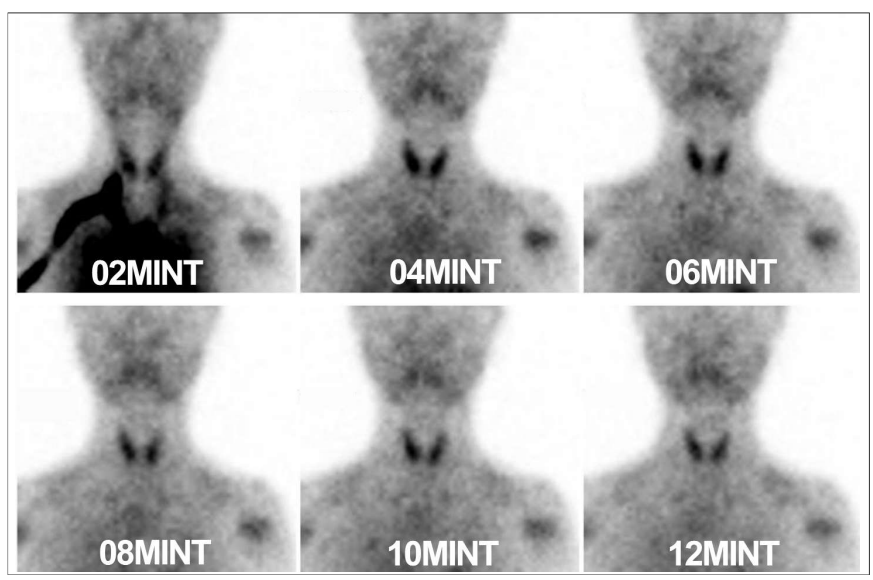

Figure 4: No scintigraphic evidence of parathyroid adenoma or hyperplasia on follow-up scan after 2 months.

Table I: Relevant investigations done, by days of admission.

\begin{tabular}{l|c|c|c|c|c|l|l}
\hline Laboratory investigations & Day 1* & Day 3 & Day 5 & Day 7 & Day 9** & $\begin{array}{l}8 \text { Weeks } \\
\text { postoperative }\end{array}$ & Normal range \\
\hline Calcium & 16.3 & 14.8 & 11.4 & 12.5 & 8.3 & 9.8 & $8.5-10.5 \mathrm{~g} / \mathrm{dL}$ \\
lonized calcium & 8.49 & 8.02 & 6.76 & 7.39 & 4.95 & 5.44 & $4.56-5.40 \mathrm{mg} / \mathrm{dL}$ \\
\hline Phosphorus & 3.1 & & & & & $3.2-6.3 \mathrm{mg} / \mathrm{dL}$ \\
Parathyroid hormone & 122.3 & & & & & $15.65 \mathrm{pg} / \mathrm{mL}$ \\
25 Hydroxy vitamin D & 69.3 & & & & & $30-40 \mathrm{ng} / \mathrm{mL}$ \\
Magnesium & 3.52 & & & & & $1.58-2.55 \mathrm{mg} / \mathrm{dL}$ \\
Prolactin & 1.82 & & & & & $3.46-19.40 \mathrm{ng} / \mathrm{mL}$ \\
ALT & 10 & & & & & $10-40 \mathrm{U} / \mathrm{L}$ \\
Alkaline phosphatase & 1118 & & & & & $40-129 \mathrm{U} / \mathrm{L}$ \\
Albumin & 3.7 & & & & & $3.5-5.0 \mathrm{G} / \mathrm{dL}$ \\
\hline
\end{tabular}

${ }^{*}$ Day of admission; $\quad{ }^{* *}$ Post surgery investigations; $\quad{ }^{* \star *}$ Normal values for male; $\quad A L T=$ Alanine aminotransferase. 


\section{DISCUSSION}

PHPT is often recognised on the biochemical evaluation or during the screening for decreased bone mass. PHPT mostly presents in patients from the age of 45 to 75 years.2,3 PHPT is rare among children and manifests either as skeletal bone deformities or nephrolithiasis. 4,5 Skeletal deformities such as genu valgum as a presenting feature of PHPT is uncommon. However, it was one of the skeletal deformities of our patient. PHPT is most often due to a parathyroid adenoma followed by glandular hyperplasia, radiation therapy, MEN, and hereditary conditions. ${ }^{6}$ Drug history is also important since thiazide and lithium can also cause an elevated calcium level. ${ }^{7}$ The present patient denied the drug history other than calcium and vitamin D supplements.

The most common clinical presentation of PHPT is asymptomatic hypercalcemia. In children, clinical presentation is variable with signs and symptoms of fatigue, generalised body weakness, bone pain, back ache, bone fracture, swollen joints, nausea, vomiting, constipation, loss of appetite, depression, polyuria, polydipsia, nocturia, memory loss and associated conditions of hematuria, weight loss and ulcers, which mimic the clinical features of hypercalcemia. 8 In order to differentiate it from secondary hyperparathyroidism, there is hypocalcemia and hyperphosphatemia in later as compared to the former.

The diagnosis of PHPT is suspected whenever there is finding of a raised serum calcium level. To confirm it, intact PTH should be measured along with serum calcium. 25-OH-Vitamin D level should be checked in all patients suspected of PHPT. The Endocrine Society guidelines recommend that in all patients suspected of PHPT, a serum 25-hydroxy vitamin D level should be assessed to rule out vitamin $D$ intoxication and correction of definitive depletion is warranted before the treatment plan. 9 In addition, PHPT may be suspected in a patient with nephrolithiasis. Once the diagnosis of PHPT is made, the underlying pathology needs to be sought. Radiological scans including ultrasound, CT and MRI of the neck are useful in the diagnosis of PHPT. $99 \mathrm{mTc}$-sestamibi parathyroid scintigraphy is considered the best preoperative localising modality for the detection of parathyroid adenomas. ${ }^{10}$

This patient had markedly increased alkaline phosphatase, low calcium, hypophosphatemia and generalised osteopenia, suggesting that the patient had rickets, for which, he received multiple calcium and vitamin $D$ supplements in the past. Work-up at the set-up showed hypercalcemia and hyperparathyroidism; whereas, sestamibi scan showed a parathyroid adenoma.

In a literature review by Dutta et al., 19 cases of PHPT were falsely diagnosed as rickets. ${ }^{11}$ Of these, 17 patients had parathyroid adenoma, one parathyroid hyperplasia, and one an ectopic parathyroid adenoma. ${ }^{11}$ In another study, 4 patients had PHPT, 3 of whom presented with bone pains mimicking rickets and 1 with short stature and generalised body aches associated with asymptomatic hypercalcemia. ${ }^{12}$

This case report highlights that PHPT can masquerade the clinical features of rickets. In a patient with skeletal deformities of rickets, no improvement in the clinical spectrum following calcium and vitamin D supplementation; and hypercalcemia at presentation the index of suspicion for the diagnosis of PHPT should be kept high.

In conclusion, parathyroid adenomas, although rare in children, is an important cause of PHPT in children and adolescents.

It is, therefore, necessary to keep this condition in the differential diagnosis in children and adolescents presenting with clinical features of rickets, but showing no response to vitamin $\mathrm{D}$ and calcium supplementation.

\section{PATIENT'S CONCENT:}

Written consent was taken from the patient and the father.

\section{CONFLICT OF INTEREST:}

Authors declared no conflict of interest.

\section{AUTHORS' CONTRIBUTION:}

KAK: Primary consultant, and advisor on the case report. SUQ: Writer, literature searcher, and final drafter of the case report.

\section{REFERENCES}

1. Silverberg SJ, Bilezikian JP. The diagnosis and management of asymptomatic primary hyperparathyroidism. Nat Clin Pract Endocrinol Metab 2006; 2:494-503.

2. Griebeler ML, Kearns AE, Ryu E, Hathcock MA, Melton III LJ, Wermers RA. Secular trends in the incidence of primary hyperparathyroidism over five decades (1965-2010). Bone 2015; 73:1-7.

3. Yeh MW, Ituarte PH, Zhou HC, Nishimoto S, Amy Liu IL, Harari A. et al. Incidence and prevalence of primary hyperparathyroidism in a racially mixed population. $J$ Clin Endocrinol Metab 2013; 98:1122-9.

4. Sneider MS, Solorzano CC, Montano RE, Anello C, Irvin III GL, Lew Jl. Sporadic primary hyperparathyroidism in young individuals: Different diseases and treatments. J Surg Res 2009; 155:100-3.

5. Heath III H, Hodgson SF, Kennedy MA. Primary hyperparathyroidism: Incidence, morbidity, and potential economic impact in a community. N Engl J Med 1980; 302:189-93.

6. McDonald DK, Parman L, Speights Jr VO. Primary hyperparathyroidism due to parathyroid adenoma. Radiographics 2005; 25:829-34.

7. Sato K. Drug-induced hypercalcemia. Clin Calcium 2006; 16:67-72.

8. Shah VN, Bhadada SK, Bhansali A, Behera A, Mittal BR, Bhavin V. Influence of age and gender on presentation of 
symptomatic primary hyperparathyroidism. J Postgrad Med 2012; 58:107-11.

9. LeBlanc ES, Zakher B, Daeges M, Pappas M, Chou R. Screening for vitamin $D$ deficiency: A systematic review for the US preventive services task force. Ann Intern Med 2015; 162: 109-22.

10. Giordano A, Rubello D, Casara D. New trends in parathyroid scintigraphy. Eur J Nucl Med 2001; 28:1409-20.
11. Dutta D, Kumar M, Das RN, Datta S, Biswas D, Ghosh S, et al. Primary hyperparathyroidism masquerading as rickets: Diagnostic challenge and treatment outcomes. J Clin Res Pediatr Endocrinol 2013; 5:266-9.

12. Ganie MA, Raizada N, Chawla H, Singh AK, Aggarwala S, Bal CS. Primary hyperparathyroidism may masquerade as ricketsosteomalacia in vitamin D repleted children. J Pediatr Endocrinol Metab 2016; 29:1207-13. 